\title{
Impact of diffusion weighted magnetic resonance imaging in diagnosis of cervical cancer
}

\author{
Sahar Mahmoud Abd elsalam(D, Omnia Mokhtar², Lamia Adel ${ }^{3}$, Reda Hassan², Manal Ibraheim ${ }^{4}$ and Amro Kamal ${ }^{5}$
}

\begin{abstract}
Background: Cervical cancer is a widespread cancer among women worldwide. In this study, we evaluated the role of diffusion weighted magnetic resonance imaging (DWI-MRI) in diagnosis of cervical carcinoma with pathological diagnosis taken as the reference. The study included 70 patients in whom cancer cervix had been suspected clinically or by transvaginal U/S and confirmed by biopsy. The control group consisted of 40 patients with a normal cervix who performed MRI for other pelvic disorders. Pelvic MR with DWI and dynamic contrast enhanced MRI (DCE-MR) were done for all patients.

Results: Both DW-MRI and DCE-MRI revealed the same sensitivity, specificity, PPV, and accuracy; 100\%, 50\%, 97\%, and $97 \%$, respectively. The mean ADC value for malignant lesions was $\left(0.82 \times 10^{-3} \pm 0.1 \mathrm{SD} \mathrm{mm} / \mathrm{s}\right)$. While the mean ADC value in the control group is $\left(1.56 \times 10^{-3} \mathrm{~mm}^{2} / \mathrm{s}\right)$. ADC value of $\left(1.07 \times 10^{-3} \mathrm{~mm}^{2} / \mathrm{s}\right)$ is a cut-off between normal cervical tissue\& malignant cervical lesion with a sensitivity $97 \%$ and specificity $95.5 \%$

Conclusion: Each of the (DW-MRI) and (DCE-MRI) sequences when added to the non-contrast MRI sequences in the estimation of cancer cervix had elicited the same sensitivity, specificity, and accuracy. DW-MRI was significantly beneficial in terms of diagnostic performance. For patients who cannot receive contrast medium, dynamic contrastenhanced imaging can be replaced by DWI. The ADC value in case of cervical cancer was significantly lower than in the non-affected cervical tissue.
\end{abstract}

Keywords: Cervical carcinoma DW-MRI, ACD value, DCE-MRI

\section{Background}

Cervical cancer is the fourth most widespread cancer in women worldwide, and the second most common in lowand middle-income countries. In 2018, there were an estimated 569,847 new cases universally annually. More than $85 \%$ of these cases arise in developing countries [1].

In Egypt, cervical cancer is the thirteenth most frequent cancer among women in Egypt and the tenth most frequent cancer amongst women between 15 and 44 years of age [2].

MRI is an ideal non-invasive technique and has betterquality compared to other imaging modalities in the evaluation of pelvic abnormalities. MR imaging represents the single most valuable modality for detection of

\footnotetext{
*Correspondence: shahdsahar@yahoo.com; saharmahmoud575@gmail.com ${ }^{1}$ Radiology Department, Faculty of Medicine, Beni-suef University, Beni Suef, Egypt

Full list of author information is available at the end of the article
}

primary tumor, nodal involvement and local spread. It is also the best modality for showing recurrent disease and monitoring therapeutic response [3].

MRI is superior to clinical examination alone for correctly evaluating cervical carcinoma stage. The recent 2018 International Federation of Gynecology and Obstetrics Federation (FIGO) staging system of cervical cancer emphasizes the usefulness of imaging, and includes imaging evaluation, when available, in addition to clinical examination as part of clinical staging [4]. MRI can accurately determine tumor location (exophytic or endocervical), tumor size, depth of stromal invasion, and extension into the lower uterine segment. This is of particular importance for the choice of treatment [3].

Diffusion-weighted imaging (DWI) is a functional MRI sequence which is increasingly employed for tumor diagnostics. It carries the potential to improve tissue characterization when findings are interpreted together with conventional 
Table 1 The correlation between the number of cases diagnosed as benign or malignant by DCE-MR imaging, DWI, and pathological diagnosis

\begin{tabular}{llll}
\hline & Malignant & Benign & Total \\
\hline DCE-MRI & $68(97.1 \%)$ & $2(2.9 \%)$ & $70(100 \%)$ \\
DWI-MRI & $68(97.1 \%)$ & $2(2.9 \%)$ & $70(100 \%)$ \\
Pathology & $66(94.3 \%)$ & $4(5.7 \%)$ & $70(100 \%)$ \\
\hline
\end{tabular}

MR imaging sequences [5]. Moreover, DWI permits quantitative evaluation of diffusion properties based on the calculated apparent diffusion coefficient (ADC), which is a function of the exponential reduction in tissue signal intensity with increasing diffusion-weighting ( $b$ values) [6].

The aim of our study was to evaluate the additive role of diffusion weighted magnetic resonance imaging (MRIDWI) in assessment of cervical carcinoma, with pathological diagnosis taken as the reference.

\section{Methods}

\section{Patient population}

A case-control study was conducted on 70 consecutive women (case group); in whom cervical cancer had been suspected clinically or by transvaginal U/S and confirmed histopathologically in the period of January 2014 to June 2016. The control group consisted of 40 women age matched in whom cervical cancer had not been suspected and MRI was performed due to other pelvic diseases. The patient's age ranged from 38 to 82 years old. Our study was approved from the ethical committee of our institution. Data were collected after obtaining informed written consent of all cases.

\section{MRI imaging protocol}

MR imaging was carried out using a $1.5 \mathrm{~T}$ scanner (Achieva, Philips medical system) using pelvic phased-array Torso coil with the patient in the supine position. All cases were asked to check their creatinine level before the examination to avoid renal insult caused by contrast media administration. MR protocol included

- Non-contrast MRI images including axial T1weighted (TR/TE, 500/10 ms), axial T2-weighted (TR/TE, 3300/100 ms), slice thickness $6 \mathrm{~mm}$, gap $1 \mathrm{~mm}$, and FOV 32-42 cm. Matrix $256 \times 256$. coronal and sagittal T2-weighted, slice thickness 8-10 $\mathrm{mm}$, gap $1 \mathrm{~mm}$, and FOV $40-50 \mathrm{~cm}$. Matrix $256 \times 256$.

- MR with diffusion weighted imaging (DWI) was obtained in the axial plane preceding administration of contrast medium by using a single shot echo-planar imaging sequence, with $b$ values 0,300 , and 600. TR/TE, 5000/70. Slice thickness $6 \mathrm{~mm}$, gap $1 \mathrm{~mm}$, and FOV $36 \mathrm{~cm}$. Matrix, $128 \times 128$.

- Dynamic contrast-enhanced MRI (DCE-MR): post-contrast T1 fat sat THRIVE (high-resolution isotropic volume examination) images were acquired instantly after administration of $0.1 \mathrm{mmol} / \mathrm{kg}$ of body weight (maximum, $20 \mathrm{~mL}$ ) gadolinium (manual injection). This was followed by injection of $20 \mathrm{~mL}$ of normal saline flushing the tube. Images were acquired serially at $0,30,60,90$, and $120 \mathrm{~s}$.
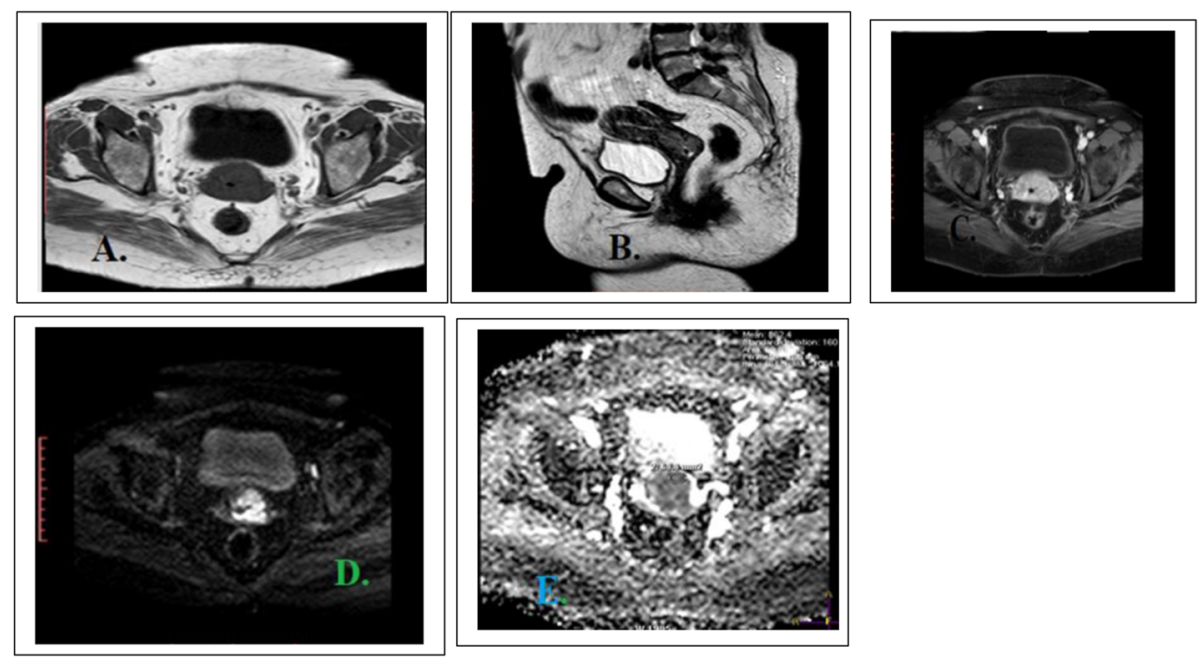

Fig. 1 Eighty-two-year-old female patient complaining of post-menopausal bleeding. a Axial T1WI revealed isointense cervical soft tissue mass lesion measuring $4 \times 3.5 \times 3 \mathrm{~cm}$ in (AP, transverse, CC) dimensions. b Sagittal T2Wl showed relatively hyper intense signal. c Post-contrast axial image with heterogeneous enhancement. $\mathbf{d}$ High signal on DWI and low signal on the corresponding ADC maps (e). ADC value was $(0.831 \times$ $10^{-3} \mathrm{~mm}^{2} / \mathrm{s}$ ). Finding suggestive of malignant lesion. Pathology revealed papillary adenocarcinoma 

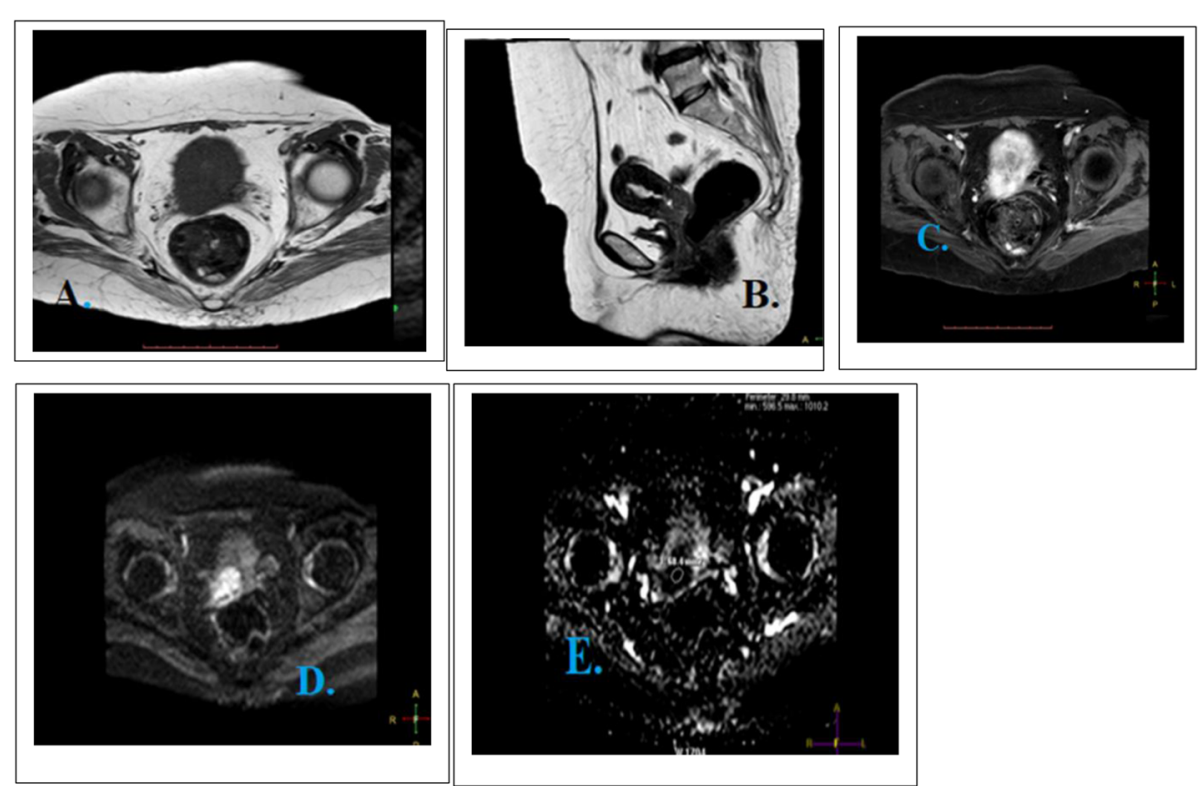

Fig. 2 Fifty-five-year-old female patient complaining of post-menopausal bleeding. a Axial T1WI revealed low signal cervical canal circumferential thickening measuring $3 \times 2.7 \times 3 \mathrm{~cm}$ in (AP, transeverse, CC) dimensions. b Sagittal T2Wl showed intermediate signal. c Post-contrast axial image with heterogeneous enhancement. $\mathbf{d}$ High signal on DWI and low signal on the corresponding ADC maps (e). ADC value was $\left(0.831 \times 10^{-3}\right.$ $\mathrm{mm}^{2} / \mathrm{s}$ ). Finding suggestive of malignant lesion. Pathology revealed non-keratinizing squamous cell carcinoma
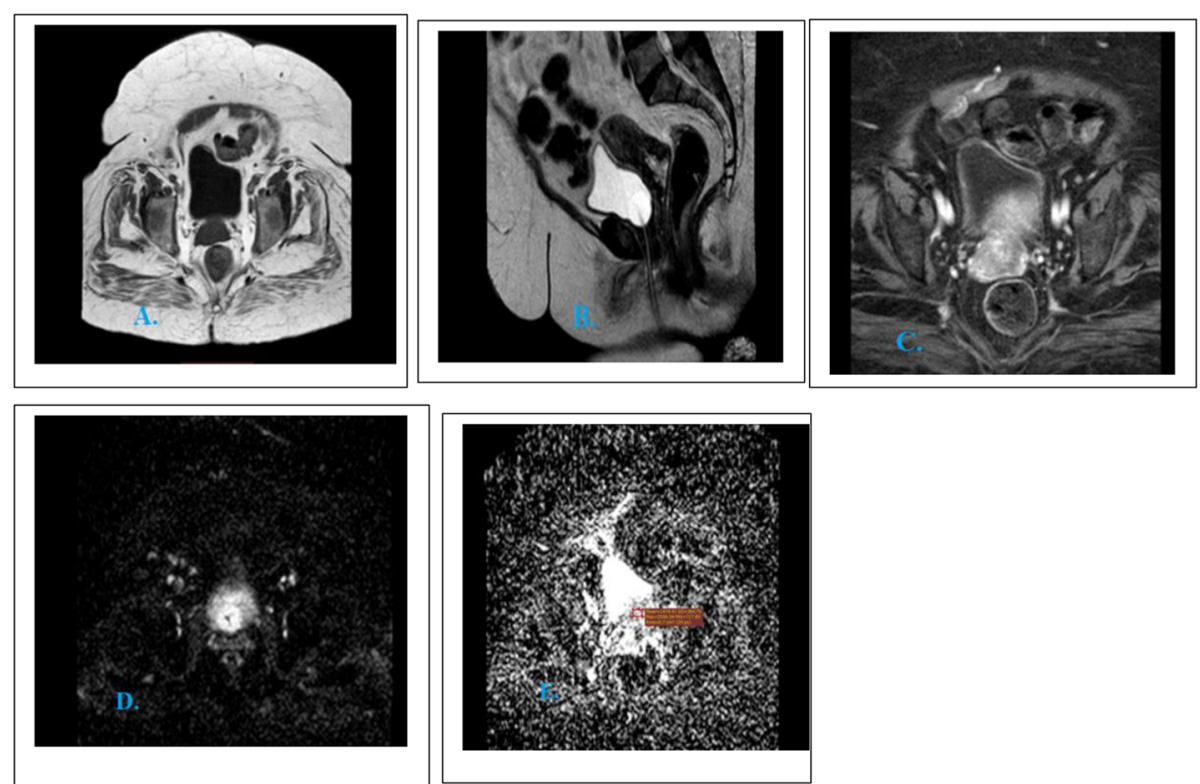

Fig. 3 Seventy-year-old female patient presenting with post-menopausal bleeding. a-c Axial T1WI, sagittal T2WI and post-contrast axial image revealed the presence of enhancing soft tissue lesion distended the cervical canal with intact surrounding serosa, measuring $2 \times 2 \times 3 \mathrm{~cm}$ in (AP, transverse, CC) dimensions, low signal intensity on T1 and high signal intensity on T2, post-contrast heterogeneous enhancement. High signal on $\mathrm{DWI}(\mathbf{d})$, and high signal on the corresponding ADC maps $(\mathbf{e})$. ADC value was $\left(1.474 \times 10^{-3} \mathrm{~mm}^{2} / \mathrm{s}\right)$. Finding suggestive of benign lesion. Pathology revealed: chronic cervicitis 

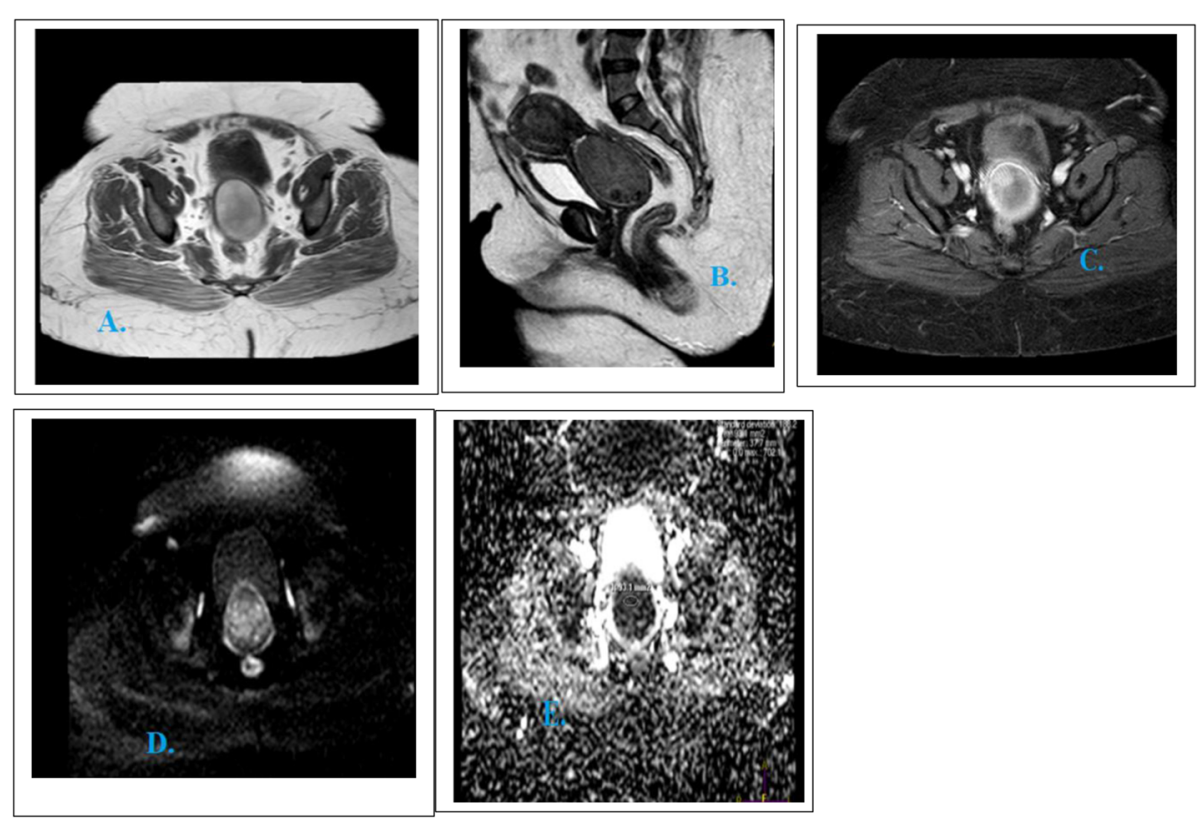

Fig. 4 Female patient 57 presenting with post-menopausal bleeding. a-c Axial T1WI, sagittal T2WI, and post-contrast axial image revealed the presence of a rather well defined heterogeneous soft tissue mass lesion filling the endocervical canal measuring $5.8 \times 5.2 \times 8 \mathrm{~cm}$ in (AP, transverse, CC) dimensions, with blurring of posterior low signal intensity serosa of the cervix denoting possibility of parametrial invasion. It elicits intermediate signal on T2 and hyper intense signal on T1 with enhancing mural nodule. High signal on DWl (d) and low signal on the corresponding ADC maps (e). ADC value was $\left(0.831 \times 10^{-3} \mathrm{~mm}^{2} / \mathrm{s}\right)$. Finding suggestive of malignant lesion. Pathology revealed leiomyoma with chronic cervicitis
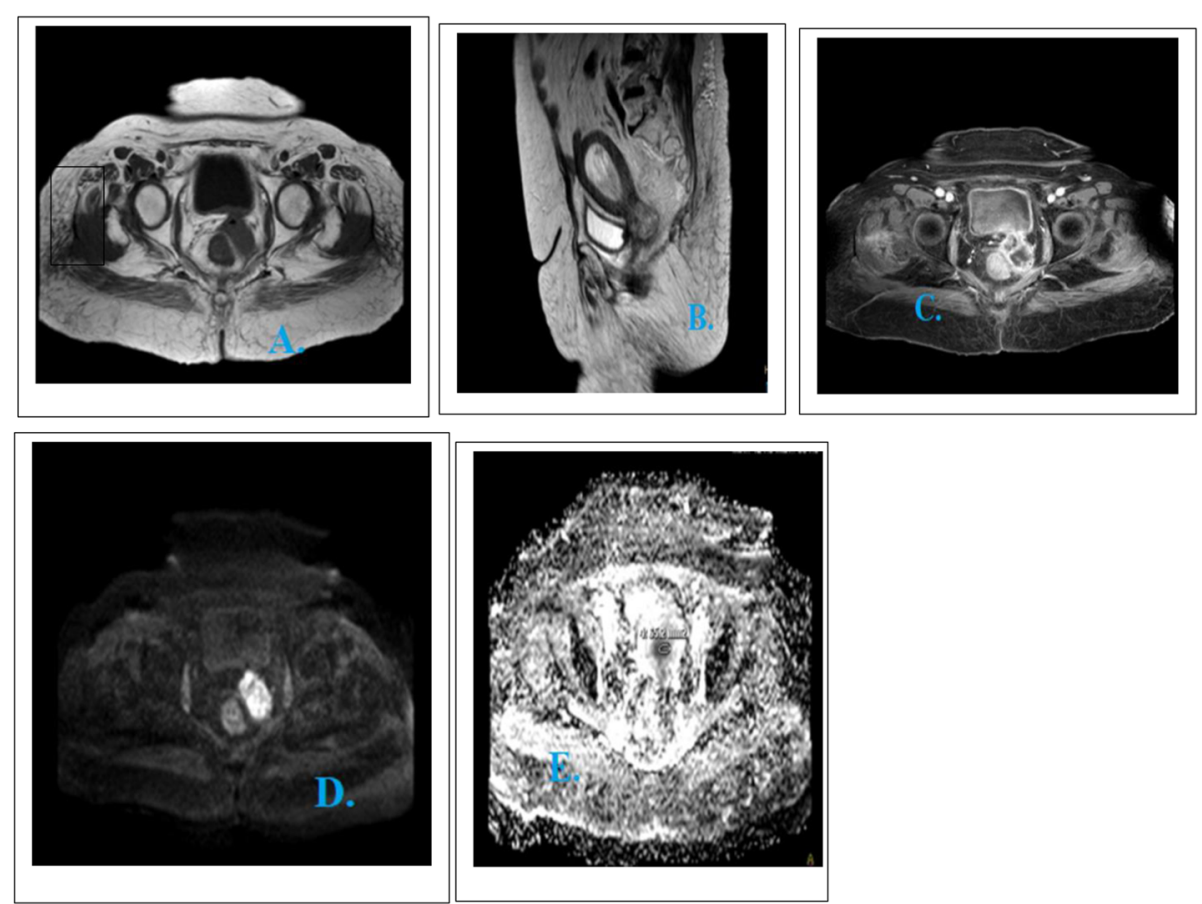

Fig. 5 Forty-five-year-old female patient presenting with irregular vaginal bleeding. a-c Axial T1WI, sagittal T2WI, and post-contrast axial image revealed the presence of ill-defined heterogeneously enhancing exophytic soft tissue mass lesion centered upon and infiltrating the cervix measuring $6 \times 4 \times 4.7 \mathrm{~cm}$ in (AP, transverse, CC) dimensions. It is also seen inseparable from the left posterior urinary bladder wall with smudging of surrounding fat planes. It elicits isointense signal on T1 and hyper intense signal on T2. High signal on DWI (d) and low signal on the corresponding ADC maps (e). $A D C$ value was $0.644 \times 10^{-3} \mathrm{~mm}^{2} / \mathrm{s}$. Finding suggestive of malignant lesion. Pathology revealed non-keratinizing squamous cell carcinoma 
Table 2 The diagnostic performance of DCE-MRI in diagnosis of cervical carcinoma

\begin{tabular}{lll}
\hline DCE-MRI & Pathological diagnosis & \\
\cline { 2 - 3 } & $\begin{array}{l}\text { Benign } \\
n=4\end{array}$ & $\begin{array}{l}\text { Malignant } \\
n=66\end{array}$ \\
\hline Benign & $2(\mathrm{TN})$ & $0(\mathrm{FN})$ \\
Malignant & $2(\mathrm{FP})$ & 66 (TP) \\
Sensitivity & $100 \%$ & \\
Specificity & $50 \%$ & \\
PPV & $97 \%$ & \\
NPV & $100 \%$ & \\
Accuracy & $97 \%$ & \\
\hline
\end{tabular}

\section{MRI imaging analysis}

- MR images were analyzed by two experienced radiologists in body MRI (15 and 10 years' experience). They reach the final diagnosis in consensus. MR images were evaluated for the following:

- $>$ MRI appearance of the tumor as regard its size, signal intensity, and enhancement.

- $>$ Involvement of other pelvic organs.

- $>$ Occurrence of infiltrated pelvic or para aortic lymph nodes and presence of peritoneal or omental deposits or hydronephrosis.

- $>$ Presence of ascites.

\section{Staging analysis}

A combination of T2-weighted images, DWI-MR imaging sequences, and dynamic post-contrast MR imaging were used in staging of cervical carcinoma. The MRI staging followed the FIGO staging analysis.

\section{Interpretation of DWI}

\section{Qualitative analysis}

Benign lesions displayed low signal intensity on DW images and high signal in the corresponding ADC maps (facilitated diffusion). Malignant lesions demonstrated

Table 3 The diagnostic performance of DWI in diagnosis of cervical carcinoma

\begin{tabular}{lll}
\hline DWI & \begin{tabular}{l} 
Pathological diagnosis \\
\cline { 2 - 3 }
\end{tabular} & $\begin{array}{l}\text { Benign } \\
n=4\end{array}$ \\
\hline Benign & $2(\mathrm{TN})$ & $\begin{array}{l}\text { Malignant } \\
n=66\end{array}$ \\
Malignant & $2(\mathrm{FP})$ & 66 (TP) \\
Sensitivity & $100 \%$ & \\
Specificity & $50 \%$ & \\
PPV & $97 \%$ & \\
NPV & $100 \%$ & \\
Accuracy & $97 \%$ & \\
\hline
\end{tabular}

Table 4 Sensitivity, specificity, PPV, NPV, and accuracy of (MRI+DWI) compared to (conventional MRI+ DCE-MRI)

\begin{tabular}{lll}
\hline & Conventional MRI+ DWI & Conventional MRI+DCE-MRI \\
\hline Sensitivity & $100 \%$ & $100 \%$ \\
Specificity & $50 \%$ & $50 \%$ \\
accuracy & $97 \%$ & $97 \%$ \\
PPV & $97.06 \%$ & $97.06 \%$ \\
NPV & $100 \%$ & $100 \%$ \\
$P$ value & 0.057 & 0.057 \\
\hline
\end{tabular}

high signal intensity on DW images and low signal intensity on ADC maps (restricted), avoiding reduced signal intensity areas which could indicate necrotic areas.

\section{Quantitative analysis}

Regarding the quantitative analysis of DWI, ADC maps were generated. Tumor regions of interest (ROIs) were manually identified, which were then automatically calculated on the work station to get the mean $\mathrm{ADC}$ values $\left(\times 10^{-3} \mathrm{~mm}\right)$.

\section{Statistical analysis}

Data analysis was performed using SPSS v. 22 (Statistical Package for Social science) for Windows. Quantitative variables were described as mean, standard deviation (SD), minimum, and maximum. Diagnostic tests including sensitivity, specificity, PPV, NPV, and accuracy were calculated using MedCalc statistical software version 18.10.2. Pathological data as the gold standard in our study was considered. Receiver operating characteristic (ROC) curve was used to predict malignant cervical lesions by using ADC values. The significance of the results was assessed in the form of $P$ value that was differentiated into non-significant when $P$ value $>0.05$, significant when $P$ value $\leq 0.05$ and highly significant when $P$ value $\leq 0.01$.

\section{Results}

The study group consisted of 70 patients in whom cervical cancer had been suspected clinically or by transvaginal U/S and confirmed by biopsy. The control group consisted of 40 patients in whom cervical cancer had not been suspected and MRI was performed because of other pelvic diseases.

The patients' ages ranged between 38 and 82 years (mean age 59.4). The clinical presentations were postmenopausal bleeding $(32 / 70,45.7 \%)$, vaginal bleeding with discharge $(16 / 70,22.8 \%)$, and vaginal bleeding with pain $(22 / 70,31.4 \%)$. The size of the cervical masses ranged between $2-5 \mathrm{~cm}$ in $14 / 70(20 \%)$ and more than 5 $\mathrm{cm}$ in $56 / 70$ (80\%).

Regarding the signal intensity of the cervical lesions at T1WIs, hypointense T1 signal was seen in $34 / 70(48.5 \%)$ cases, iso-intense signal in $28 / 70$ 
Table 5 The ADC values in malignant cervical lesions and the control group

\begin{tabular}{lllllll}
\hline ADC value & Mean & SD & Median & Minimum & Maximum & $P$ value \\
\hline Malignant lesions & 0.82 & 0.18 & 0.83 & 0.42 & 1.45 & $<0.001$ \\
The control group & 1.56 & .22 & 1.56 & 1.23 & 2.04 & $<0.001$ \\
\hline
\end{tabular}

(40\%) cases, hyper-intense T1 signal was seen in 6/ $70(8.5 \%)$ cases, heterogeneous signal intensity in $2 /$ $70(2.8 \%)$. T2WIs showed hyper-intense signal in $62 / 70(88 \%)$ cases and intermediate signal in $2 / 70$ $(2.8 \%)$ cases and heterogeneous signal intensity in $6 / 70(8.5 \%)$.

Dynamic contrast-enhanced MRI (DCE-MRI) showed heterogeneous post-contrast enhancement in 40/70, (57\%), homogenous post-contrast enhancement in 20/70 (28.5\%) and mural enhancement in 4/70 (9\%) cases. Diffusion weighted images showed high signal (restricted diffusion) of cervical lesions in 68/70 (97.1\%) cases.

By DCE-MRI, 68 (97.1\%) cases were diagnosed as malignant cervical lesions and 2 (2.9\%) cases were diagnosed as benign lesions. DWI-MRI also had the same results as DCE-MRI. Pathological examination revealed 66 (94.3\%) cases diagnosed as malignant cervical lesions and 4 (5.7\%) cases benign lesions (Table 1) (Figs. 1, 2, 3, 4, and 5).

The results of histopathological examination were compared to conventional MRI, DWI-MRI, and DCEMRI images to detect sensitivity, specificity, accuracy, $\mathrm{PPV}$, and NPV in diagnosis of malignant cervical lesions (Tables 2, 3, and 4).

The ADC values were calculated for the malignant cervical lesions and the control group. The mean ADC values for malignant lesions was $0.82 \times 10^{-3} \pm 0.1 \mathrm{SD} \mathrm{m^{2 }} / \mathrm{s}$ while the mean $\mathrm{ADC}$ value in the control group was $1.56 \times 10^{-3}$ $\mathrm{mm}^{2} / \mathrm{s}$ (Table 5). Quantitative analysis of the ADC values in different histopathological types of cervical malignant lesions and the detected benign lesions showed no significant statistical difference (Table 6).

Results of ROC analysis of ADC values differentiating between cervical carcinoma and normal cervical tissue are shown in Table 7. The area under the curve was 0.942 with a significant $P$ value of 0.001 . ADC value of $1.07 \times 10^{-3} \mathrm{~mm}^{2} / \mathrm{s}$ was the cut off between normal cervical tissue and malignant cervical lesions with sensitivity 97\% and specificity $95.5 \%$ (Fig. 6).

\section{Discussion}

Cervical cancer is a major cause of morbidity and mortality worldwide in spite of advances in screening and prevention. The oncologic challenges include early detection, appropriate treatment, and interventions to control the disease. The 2018 FIGO committee has encouraged the use of advanced imaging modalities, including MR imaging, to increase the accuracy of staging and help guide treatment, radiation treatment planning/ monitoring, and detection of recurrence [4].

Extensive literature is found supporting the role of MRI in the pretreatment evaluation of patients with cervical cancer. MRI provided detailed findings being an advanced multi-planar multi-parametric modality that made staging and consequently management much easier procedure in a way that overweighs its high cost [7-10].

On contrast enhanced MRI, cervical cancer presents high signal relative to the low signal of the cervical stroma. Typically, cervical tumors enhance avidly in the early dynamic phase compared to the slight enhancement of the normal cervical epithelium and stroma, permitting distinction of recurrent tumors from radiation fibrosis [11].

In the present study, heterogeneous contrast enhancement was found in 57\% of cases. Each of the DWI and DCE-MRI sequences when added to the non-contrast MRI sequences in the estimation of cancer cervix had

Table 6 Quantitative (ADC) values in different histopathological types

\begin{tabular}{|c|c|c|c|c|c|c|c|}
\hline & & \multirow[b]{2}{*}{ Number of cases (\%) } & \multicolumn{5}{|c|}{ Quantitative (ADC) } \\
\hline & & & Mean & SD & Median & Minimum & Maximum \\
\hline \multirow[t]{9}{*}{ Pathology } & chronic cervicitis & $1(3 \%)$ & 1.16 & 0.0 & 1.16 & 1.16 & 1.16 \\
\hline & endometrial adenocarcinoma & $3(8.5 \%)$ & 0.84 & 0.0 & 0.84 & 0.84 & 0.84 \\
\hline & Keratinized squamous cell carcinoma & $10(28.5 \%)$ & 0.84 & 0.09 & 0.88 & 0.69 & 0.96 \\
\hline & Leiomyoma\&chronic cervicitis & $1(3 \%)$ & 0.47 & 0.0 & 0.47 & 0.47 & 0.47 \\
\hline & Mixed Mullerian carcinoma & $2(6 \%)$ & 0.85 & 0.0 & 0.85 & 0.85 & 0.85 \\
\hline & Non-keratinized squamous cell carcinoma & $8(23 \%)$ & 0.76 & 0.15 & 0.78 & 0.42 & 0.98 \\
\hline & Papillary adenocarcinoma & $3(8.5 \%)$ & 0.87 & 0.14 & 0.89 & 0.64 & 0.99 \\
\hline & Undifferentiated carcinoma & $4(11 \%)$ & 0.76 & 0.01 & 0.76 & 0.76 & 0.77 \\
\hline & Villoglandular adenocarcinoma & $3(8.5 \%)$ & 0.80 & 0.0 & 0.80 & 0.80 & 0.80 \\
\hline
\end{tabular}


Table 7 The cut off between normal cervical tissue and malignant cervical lesion

\begin{tabular}{|c|c|c|c|c|c|c|}
\hline \multirow{2}{*}{$\begin{array}{l}\text { Area } \\
\text { under } \\
\text { curve }\end{array}$} & \multirow{2}{*}{$\begin{array}{l}P \\
\text { value }\end{array}$} & \multicolumn{2}{|c|}{ 95\% confidence interval for AUC } & \multirow{2}{*}{$\begin{array}{l}\text { Cutoff } \\
\text { value } \\
\left(\mathrm{mm}^{2} /\right. \\
\mathrm{s})\end{array}$} & \multirow{2}{*}{$\begin{array}{l}\text { Sensitivity } \\
(\%)\end{array}$} & \multirow{2}{*}{$\begin{array}{l}\text { Specificity } \\
(\%)\end{array}$} \\
\hline & & Lower bound & Upper bound & & & \\
\hline .942 & $<0.001$ & .854 & 1.030 & 1.0705 & 97 & 95.5 \\
\hline
\end{tabular}

elicited the same sensitivity value (100\%), specificity (50\%), and accuracy (97\%).

Malignant cervical tissue demonstrates restricted diffusion and hence reduced ADC values when compared to normal tissue. DWI and ADC maps allow differentiation of benign from malignant zones of cervix with high sensitivity and specificity [12].

The current study, DWI-MRI showed sensitivity $100 \%$, specificity $50 \%$, accuracy $97 \%$, PPV $97 \%$, and NPV $100 \%$, compared to DCE-MRI which reported sensitivity $100 \%$, specificity $50 \%$, accuracy $97 \%$, PPV $97 \%$, and NPV $100 \%$. The low percentage of the specificity in this study was due to the low number of the true negative patients. This is in accordance to Chen et al. [13] who found that the sensitivity and specificity of DW-MRI for tumor detection were $100 \%$ and $84.8 \%$, respectively.

The current study results are similar to the study carried out by Kuang et al. [14] that included 75 cervical carcinoma and 47 benign cervical lesions (25 cervical leiomyoma, 22 cervical polyps). In their results, DWI-MRI was significantly better than routine MRI and revealed high accuracy (0.95); the diagnostic performance was not significantly different between DWI-MRI and DCE-MRI.

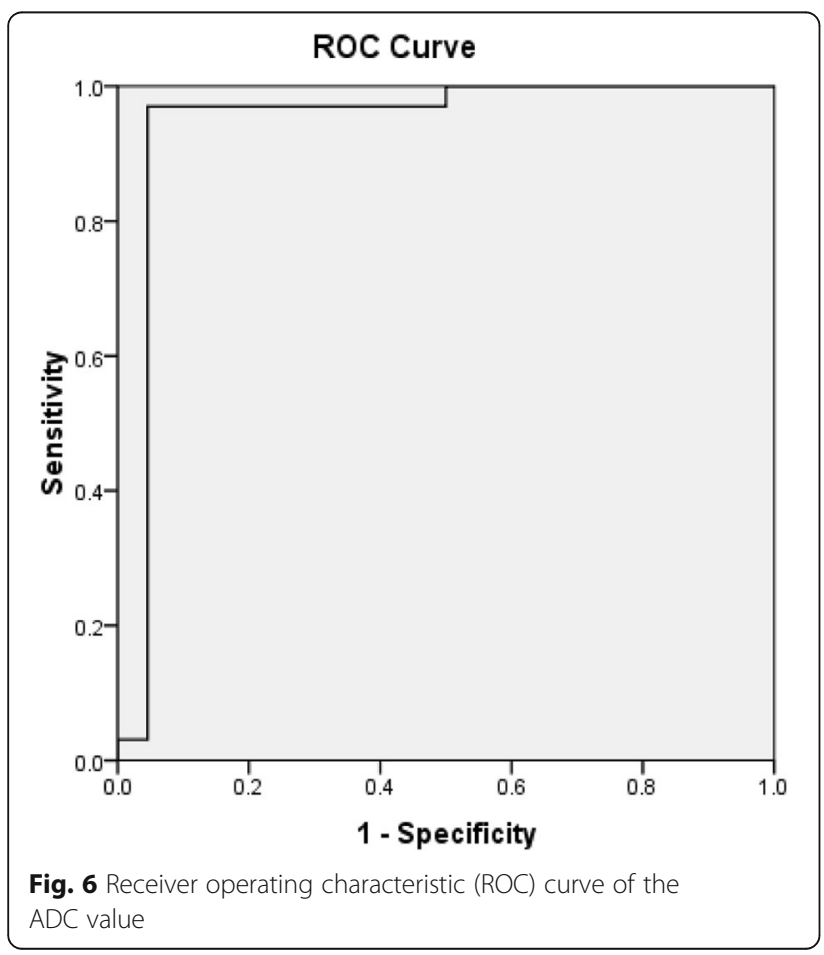

In their study, DWI-MRI displayed 95\% sensitivity, 96\% specificity, 95\% accuracy, 97\% PPV, and 92\% NPV compared to DCE-MRI that displayed sensitivity $96 \%$, specificity $96 \%$, accuracy $96 \%$, PPV $97 \%$, and NPV $94 \%$.

In the present study, the mean ADC value for malignant lesions was $0.82 \times 10^{-3} \pm 0.1 \mathrm{SD} \mathrm{mm}^{2} / \mathrm{s}$ ), while the mean ADC value in the control group was $1.56 \times 10^{-3}$ $\mathrm{mm}^{2} / \mathrm{s}$ ). The ADC value of $1.07 \times 10^{-3} \mathrm{~mm}^{2} / \mathrm{s}$ was the cut off between benign and malignant cervical lesion by sensitivity $97 \%$, specificity $95.5 \%$, and $P$ value $\leq 0.001$. A combination of increased extracellular tortuosity and the ratio of intra- to extracellular water fraction may be the best biological explanation for the decreased ADCs in the cancer tissues [15].

Kuang et al. [14] found that the mean ADC values for cervical carcinoma were $0.916 \times 10^{-3} \pm 0.15 \mathrm{SD} \mathrm{mm} / \mathrm{s}$, for cervical leiomyoma $1.396 \times 10^{-3} \pm 0.15 \mathrm{SD} \mathrm{m^{2 }} / \mathrm{s}$ and for cervical polyp $\left(1.426 \times 10^{-3} \pm 0.11 \mathrm{SD} \mathrm{m^{2 }} / \mathrm{s}\right.$. There were significant differences between the ADC values of cervical cancer and those of benign cervical lesions (leiomyoma and cervical polyps) at both ADC maps $(P<0.001)$; however, there was no significant difference between cervical leiomyoma and cervical polyps at both ADC maps.

Atstupènaite et al. [16] included 65 patients in whom cervical cancer had been clinically suspected and confirmed by biopsy before MRI examination. All these patients underwent pelvic MRI twice: before the chemoradiation therapy and 6 months following therapy. They found that the mean ADC value of the study group $\left(0.658 \pm 0.118 \times 10^{-3} \mathrm{~mm}^{2} / \mathrm{s}\right)$ was lower than of the control group $\left(1.171 \pm 0.143 \times 10^{-3}\right.$ $\left.\mathrm{mm}^{2} / \mathrm{s}\right)$, with $P$ value $(P=0.03)$. The ADC threshold value of $0.945 \times 10^{-3} \mathrm{~mm}^{2} / \mathrm{s}$ was defined, differentiating the cancer-affected cervical tissue from the normal tissue.

Nakmura et al. [17] studied 80 cervical cancer patients who underwent pelvic MRI within the 2 to 4 weeks before radical hysterectomy. They reported that the mean ADC value was $\left(0.852 \times 10^{-3} \mathrm{~mm}^{2} / \mathrm{s}\right)$. They also found that well differentiated tumors had higher ADC values than poorly differentiated tumors $\left(1.2 \times 10^{-3} \mathrm{~mm}^{2} / \mathrm{s}\right.$ vs. $\left.1.1 \times 10^{-3} \mathrm{~mm}^{2} / \mathrm{s}\right)(P=0.01)$.

In this study, we reported no significant difference in ADC between different histological subtypes. ADC mean of keratinized squamous cell carcinoma (K.SCC) was $0.84 \times 10^{-3} \mathrm{~mm}^{2} / \mathrm{s}$ while that of non-keratinized squamous cell carcinoma (NK.SCC) was $0.76 \times 10^{-3} \mathrm{~mm}^{2} / \mathrm{s}$ and ADC mean of papillary adenocarcinoma was $0.87 \times$ $10^{-3} \mathrm{~mm}^{2} / \mathrm{s}$. Similar results had been observed in the 
study done by Payne et al. [18] who reported lower ADCs in cases with cancer cervix compared to normal cervix with no significant difference in ADC values between different histological subtypes.

\section{Conclusion}

Each of the DW-MRI and DCE-MRI sequences when added to the non-contrast MRI sequences in the estimation of cancer cervix had elicited the same sensitivity, specificity, and accuracy. DW-MRI was significantly beneficial in terms of diagnostic performance. For patients who cannot receive contrast medium, dynamic contrast-enhanced imaging can be replaced by DWI. The ADC value in case of cervical cancer was significantly lower than in the non-affected cervical tissue.

\section{Recommendations}

Diffusion weighted MRI should be included in the routine pelvic MRI protocol. Because of time restrictions, radiologists have to make a choice between different sequences according to the patient 'clinical condition.

\section{Abbreviations}

ADC: Apparent diffusion coefficient; DCE-MRI: Dynamic contrast enhanced magnetic resonance imaging; ROC: Receiver operating curve; DWI: Diffusion weighted imaging; FIGO: International federation of obstetrics and gynecology; TVUS: Transvaginal ultrasound

\section{Acknowledgements}

Not applicable.

\section{Authors' contributions}

SA carried out statistical analysis, data collection, image analysis, drafting and editing of the paper. OM shared in image analysis and interpretation. LA shared in design and drafting of the manuscript. $\mathrm{RH}$ participated in data collection. MI participated in manuscript editing. AK carried out clinical assessment. All contributing authors have read and approved the manuscript.

\section{Funding}

No funding resources.

\section{Availability of data and materials}

All data are available at the corresponding author who has the authority to respond if there is any query.

\section{Ethics approval and consent to participate}

The study was approved from the ethical committee of Faculty of Medicine Beni-suef University (FWA00015574). Data were collected after obtaining informed written consent of all cases.

\section{Consent for publication}

All patients included in this research gave written informed consent to publish the data contained within this study.

\section{Competing interests}

The authors declare that they have no competing interests.

\section{Author details}

${ }^{1}$ Radiology Department, Faculty of Medicine, Beni-suef University, Beni Suef, Egypt. ${ }^{2}$ Radiology Department, National Cancer Institute of Cairo, Cairo, Egypt. ${ }^{3}$ Radiology Department, Faculty of Medicine, Cairo University, Cairo, Egypt. ${ }^{4}$ National Liver Institute, Menofia University, Shebeen El-Kom, Egypt. ${ }^{5}$ Surgery Department, National Cancer Institute of Cairo, Cairo, Egypt.
Received: 2 October 2019 Accepted: 21 January 2020

Published online: 28 January 2020

\section{References}

1. Bray F, Ferlay J, Soerjomataram I et al (2018) Global cancer statistics 2018: GLOBOCAN estimates of cancer incidence and mortality for 36 cancers in 185 countries. CA Cancer J Clin 68:394-424

2. Human Papillomavirus and Related Cancers, Fact Sheet. ICO HPV Information Centre Institut Català d'Oncologia; (2018). Available from: htpp:// https:// hpvcentre.net/statistics/reports/EGY_FS.pdf accessed on 2019 Oct 24].

3. Dappa E, Elger T, Hasenburg A et al (2017). The value of advanced MRI techniques in the assessment of cervical cancer: a review. Insights Imaging; 8(5):471-481. doi: 10.1007/s13244-017-0567-0. Epub 2017 Aug 21. PMID: 28828723; PMCID: PMC5621992.

4. Bhatla N, Berek JS, Cuello Fredes M et al (2019). Revised FIGO staging for carcinoma of the cervix uteri. Int J Gynaecol Obstet. 17;68(16-20):394.

5. Whittaker CS, Coady A, Culver L et al (2009) Diffusion-weighted MR imaging of female pelvic tumors: a pictorial review. Radiographics 29(3):759-774. https://doi.org/10.1148/rg.293085130

6. Bollineni VR, Kramer G, Liu Y et al (2015) A literature review of the association between diffusion-weighted MRI derived apparent diffusion coefficient and tumour aggressiveness in pelvic cancer. Cancer Treat Rev 41(6):496-502. https://doi.org/10.1016/j.ctrv.2015.03.010

7. Patel-Lippmann K, Robbins JB, Barroilhet L et al (2017). Magn Reson Imaging Clin N Am; 25(3):635-649. doi: https://doi.org/10.1016/j.mric.2017.03.007.

8. Sarabhai T, Schaarschmidt BM, Wetter A et al (2018) Comparison of (18)FFDG PET/MRI and MRI for pre-therapeutic tumor staging of patients with primary cancer of the uterine cervix. Eur J Nucl Med Mol Imaging 45(1):6776. https://doi.org/10.1007/s00259-017-3809-y

9. Bourgioti C, Chatoupis K, Rodolakis A et al (2016) Incremental prognostic value of MRI in the staging of early cervical cancer: A prospective study and review of the literature. Clin Imaging 40:72-78

10. Sala E, Rockall AG, Freeman SJ et al (2013) The added role of MR imaging in treatment stratification of patients with gynecologic malignancies: what the radiologist needs to know. Radiology 266:717-740

11. Brocker KA, Alt CD, Eichbaum M et-al (2011). imaging of female pelvic malignancies regarding MRI, CT, and PET/CT: part 1. StrahlentherOnko ; 187 (10): 611-618. doi:https://doi.org/10.1007/s00066-011-4001-0 - Pubmed citation.

12. Hoogendam JP, Klerkx WM, de Kort GA et al (2010) The influence of the bvalue combination on apparent diffusion coefficient based Differentiation between malignant and benign tissue in cervical cancer. J Magn Reson Imaging 32:376-382

13. Chen J, Zhang Y, Liang B et al (2010) The utility of diffusion-weighted MR imaging in cervical cancer. Eur J Radiol 74:e101-e106

14. Kuang F, Yan Z, Li H et al (2015). Diagnostic accuracy of diffusion-weighted MRI for differentiation of cervical cancer and benign cervical lesions at 3.0T: Comparison with routine MRI and dynamic contrast-enhanced MRI. J Magn Reson Imaging. Oct; 42(4):1094-1099.

15. Padhani A, Liu G, Koh D et al (2009) Diffusion-weighted magnetic resonance imaging as a cancer biomarker: consensus and recommendations. Neo-plasia 11(2):102-125

16. Atstupénaitè $V$, Basevičius A, Krimelis A et al (2011) Diffusion-weighted magnetic resonance imaging of cervical cancer. Acta Med Lituanica 18:139-146

17. Nakamura K, Joja I, Nagasaka T et al (2012) The mean apparent diffusion coefficient value (ADC mean) on primary cervical cancer is a predictive marker for disease recurrence. GynecolOncol 127(3):478-483

18. Payne GS, Schmidt M, Morgan VA et al (2010) Evaluation of magnetic resonance diffusion and spectroscopy measurements as predictive biomarkers in stage 1 cervical cancer. GynecolOncol 116(2):246-252

\section{Publisher's Note}

Springer Nature remains neutral with regard to jurisdictional claims in published maps and institutional affiliations. 